\title{
Segment representations with small resolution
}

\author{
Therese Biedl*
}

\begin{abstract}
A segment representation of a graph is an assignment of line segments in $2 \mathrm{D}$ to the vertices in such a way that two segments intersect if and only if the corresponding vertices are adjacent. Not all graphs have such segment representations, but they exist, for example, for all planar graphs.

In this note, we study the resolution that can be achieved for segment representations, presuming the ends of segments must be on integer grid points. We show that any planar graph (and more generally, any graph that has a so-called L-representation) has a segment representation in a grid of width and height $4^{n}$.
\end{abstract}

\section{Introduction}

The problems StRIng and SEgment have received much attention in recent history. Here, STRING refers to the problem of, given a graph $G=(V, E)$, testing whether we can assign a curve $\mathcal{C}(v)$ in $2 \mathrm{D}$ to each vertex $v$ in such a way that $\mathcal{C}(v)$ and $\mathcal{C}(w)$ intersect if and only if $(v, w)$ is an edge in the graph. Such an assignment is called a string representation. This problem is NP-hard [3] and also in NP [8]; the latter is not at all obvious because string representations sometimes require an exponential number of crossings among strings [5].

The SEGMEnT problem is a special case of the STRING problem, where we want a segment representation, i.e., $\mathcal{C}(v)$ is a line segment. The NP-hardness proof of [3] also works for segments (see [4]), so SEGmENT is also NP-hard but not known to be in NP. Indeed, the problem is unlikely to be in NP, since it was shown to be hard for the class $\exists \mathbb{R}$, the existential theory of the reals [6], and it is widely assumed that $\exists \mathbb{R}$ is a strict superset of NP. (See e.g. [9] for more on $\exists \mathbb{R}$.)

For the purposes of displaying a graph via a segment representation, we care not only about the existence, but also about the resolution required to show it. By moving endpoints slightly one can always achieve rational coordinates at the endpoints without changing the represented graph. After scaling therefore we may assume that endpoints have integer coordinates. We measure the resolution of a segment representation by the size of the grid supporting all endpoints of the segments.

${ }^{*}$ David R. Cheriton School of Computer Science, University of Waterloo, Waterloo, Ontario N2L 1A2, Canada. Supported by NSERC. The author would like to thank Daniel Gonçalvez and Cornelia Schattman for helpful input. 
Assuming $\exists \mathbb{R} \neq N$, there are some graphs that have a segment representation, but no segment-representation has exponential resolution (i.e., $O\left(c^{n}\right)$ for some constant $c$, where $n$ is the number of vertices). Namely, a segment-representation of exponential resolution can be described using $O(\operatorname{poly}(n))$ bits, and given such a description, we can check in $O(\operatorname{poly}(n))$ time whether this corresponds to a segment representation of a graph. If all graphs had a segment representation of exponential resolution, therefore SEGMENT would be in NP.

In his Ph.D. thesis in 1984 [10], Scheinermann famously asked whether every planar graph (i.e., graph that can be drawn in the plane without crossing) has a segment representation. This was proved in 2009 by Chalopin and Gonçalvez [1]; a second (independent and much more accessible) proof was given by Gonçalvez, Isenburg and Pennarun [2]. In particular, SEGMENT is not $\exists \mathbb{R}$-hard for planar graphs (the answer is simply "yes"), which raises the possibility that they always have representations of exponential resolution.

Neither of $[1,2]$ addressed the question of what resolution can be achieved. We show in this note that the representation from [2] can be modified to have resolution $4^{n}$. Our result is not specific to the construction in [2], but instead works for any graph that has an L-representation. This is a string representation of a graph where every $\mathcal{C}(v)$ has the shape of an L, i.e., it consists of a horizontal and a vertical segment that share their left/bottom endpoint. It was shown by Middendorf and Pfeiffer [7] that every graph that has an Lrepresentation also has a segment representation. The approach of [2] is hence to construct an L-representation for any planar graph and then to appeal to Middendorf and Pfeiffer's result.

It is not hard to show a bound of $O\left((2 n)^{2 n}\right)$ on the resolution achieved with the transformation by Middendorf and Pfeiffer (we review this in Section 3). Our main result is the following better bound:

Theorem 1. Any graph that has an L-representation (in particular therefore any planar graph) has a segment representation with resolution $4^{n}$.

\section{Proof of Theorem 1}

Assume that we are given an L-representation of a graph. We may assume that no endpoint of an L lies on another L, else we can lengthen its segment a bit. We may assume also that all vertices have a non-zero length horizontal and vertical segment, else we can insert a very short one. Whether two L's intersect depends only on the relative coordinates of segments; we may therefore reassign endpoint-coordinates to integers in $\{0, \ldots, 2 n-1\}$ in sorted order (breaking ties arbitrarily) and obtain the same graph. Hence assume from now on that all endpoints of all segments of L's have distinct coordinates in $\{0, \ldots, 2 n-1\}$.

It will be convenient to rotate the picture by $180^{\circ}$, so that $\mathcal{C}(v)$ becomes an $\mathrm{T}$. We describe $\mathcal{C}(v)$ by giving the four coordinates $\ell, b, r, t \in\{1, \ldots, 2 n\}$ of its bounding box $[\ell, r] \times[b, t]$; thus $\mathcal{C}(v)$ consists of the top and right side of this box.

To define $s(v)$, first let $d(v)$ by the segment from $\left(0,2^{t}\right)$ to $\left(2^{r}, 0\right)$, i.e., the downward diagonal in rectangle $\left[0,2^{r}\right] \times\left[0,2^{t}\right]$. Observe that $d(v)$ has slope $-2^{t-r}$. To obtain $s(v)$, 
intersect $d(v)$ with the halfspaces $\left\{x \geq 2^{\ell}\right\}$ and $\left\{y \geq 2^{b}\right\}$. The endpoints of $s(v)$ are hence $\left(2^{\ell}, 2^{t}-2^{t-(r-\ell)}\right)$ and $\left(2^{r}-2^{r-(t-b)}, 2^{b}\right)$, which have integral coordinates in $1, \ldots, 2^{2 n-1}$. See also Figure 1.

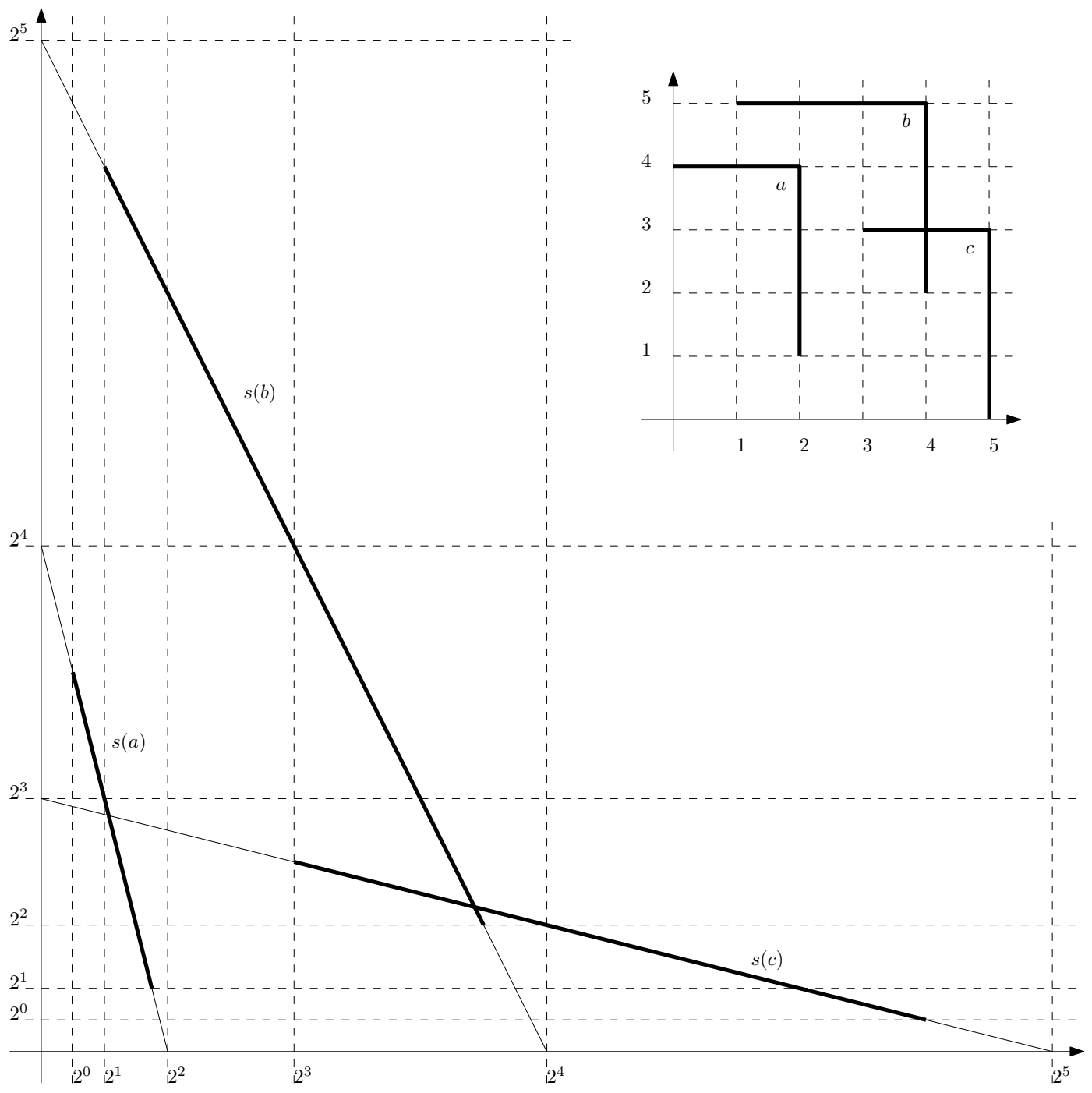

Figure 1: An L-representation (rotated) and its corresponding segment representation.

Lemma 1. If $s(v)$ and $s(w)$ intersect, then $(v, w)$ is an edge.

Proof. Let $\mathcal{C}(v)=\{\ell, b, r, t\}$ and $\mathcal{C}(w)=\{L, B, R, T\}$. After possible renaming we may assume $t>T$.

Assume that $r>R$. Walking along the $x$-axis, we encounter first $d(v)$ at $\left(2^{r}, 0\right)$ and then $d(w)$ at $\left(2^{R}, 0\right)$. Walking along the $y$-axis, we encounter first $d(v)$ at $\left(0,2^{t}\right)$, and then $d(w)$ at $\left(0,2^{T}\right)$. So the order is the same, which means that line segments $d(v)$ and $d(w)$ do not intersect, and neither can the segments $s(v)$ and $s(w)$ that lie within them. See e.g. vertices $a$ and $b$ in Figure 1. 
So we know that $r<R$ and $t>T$. If $r<L$ then $s(v)$ resides in the halfspace $\left\{x \leq 2^{r}\right\}$ and $s(w)$ resides in the halfspace $\left\{x \geq 2^{L}\right\}$ and they do not intersect. See e.g. vertices $a$ and $c$ in Figure 1. If $b>T$, then similarly $s(v)$ resides in the halfspace $\left\{y \geq 2^{b}\right\}$ while $s(w)$ resides in the halfspace $\left\{y \leq 2^{T}\right\}$, and they do not intersect.

So we know that $L<r<R$ and $b<T<t$. But now consider point $(r, T)$. This belongs to the vertical segment of $v$ by $b<T<t$ and to the horizontal segment of $w$ by $L<r<R$. So $\mathcal{C}(v)$ and $\mathcal{C}(w)$ intersect and $(v, w)$ was an edge.

Lemma 2. If $(v, w)$ is an edge, then $s(v)$ and $s(w)$ intersect.

Proof. Let $\mathcal{C}(v)=\{\ell, b, r, t\}$ and $\mathcal{C}(w)=\{L, B, R, T\}$. After possible renaming we may assume that the intersection of the curves happens between the vertical segment of $v$ and the horizontal segment of $w$. See e.g. vertices $b$ and $c$ in Figure 1. In particular, $L<r<R$ and $b<T<t$, which by integrality means $L+1 \leq r \leq R-1$ and $b+1 \leq T \leq t-1$. This immediately implies that $d(v)$ and $d(w)$ intersect (their order is different along the $x$-axis and the $y$-axis). The work is now to show that this intersection point lies within the halfspaces with which we pruned $d(v)$ and $d(w)$ to obtain $s(v)$ and $s(w)$.

We know that $d(v)$ has $y$-intercept $2^{t}$ and slope $-2^{t-r}$, while $d(w)$ has $y$-intercept $2^{T}$ and slope $-2^{T-R}$. If we let $\left(x^{*}, y^{*}\right)$ be the point of their intersection, we hence have $2^{t}-2^{t-r} x^{*}=$ $y^{*}=2^{T}-2^{T-R} x^{*}$, which implies

$$
x^{*}=\frac{2^{t}-2^{T}}{2^{t-r}-2^{T-r}} \geq \frac{2 \cdot 2^{t-1}-2^{t-1}}{2^{t-r}}=2^{r-1} .
$$

So the intersection point lies within half-space $\left\{x \geq 2^{r-1}\right\}$, which contains $\left\{x \geq 2^{L}\right\}$ since $r \geq L+1$ and $\left\{x \geq 2^{\ell}\right\}$ since $\ell<r$, hence $\ell \leq r-1$.

Symmetrically $y^{*}$ satisfies $2^{r}-2^{r-t} y^{*}=2^{R}-2^{R-T} y^{*}$ which shows that

$$
y^{*}=\frac{2^{R}-2^{r}}{2^{R-T}-2^{r-t}} \geq \frac{2^{R-1}}{2^{R-T}}=2^{T-1} .
$$

So the intersection point lies within half-spaces $\left\{y \geq 2^{b}\right\}$ and $\left\{y \geq 2^{B}\right\}$ by $T>B$ and $T>b$.

In consequence the intersection point of $d(v)$ and $d(w)$ lies on both $s(v)$ and $s(w)$ and they intersect as desired.

\section{$3\{\mathrm{I}, \mathrm{L}\}$-representations}

The result by Middendorf and Pfeiffer [7] is more general than stated before; they can create segment representations even if the input representation is an $\{\mathrm{I}, \mathrm{L}\}$-representation, i.e., vertex-curves may also be horizontally reflected Ls.

We have not been able to generalize Theorem 1 to such representations, because its proof relies on that we can distort both $x$-coordinates and $y$-coordinates exponentially. However, one can show that we can obtain a segment representation of resolution $(2 n)^{2 n}$ (specifically, its width is quite small at $2 n$, but its height is $(2 n)^{2 n}$. 
As before we will assume that the representation has distinct coordinates in $\{0, \ldots, 2 n-1\}$ and has been rotated by $180^{\circ}$. So for any vertex $v, \mathcal{C}(v)$ includes the top side of its bounding box, and it includes either the left or the right side.

To define $s(v)$, set $Q(v)$ to be the rectangle $[\ell, r] \times\left[(2 n)^{b},(2 n)^{t}\right]$, and use as $s(v)$ the diagonal of $Q(v)$ that corresponds to the ends of $\mathcal{C}(v)$. Put differently, if $\mathcal{C}$ is $\mathrm{T}$ then $s(v)$ is the downward diagonal of $Q(v)$, and if $\mathcal{C}$ is $\Gamma$ then $s(v)$ is the upward diagonal of $Q(v)$. See also Figure 2. Clearly the ends of $s(v)$ are integer grid points within the required range.

Lemma 3. If $s(v)$ and $s(w)$ intersect, then $(v, w)$ is an edge.

Proof. Let $\mathcal{C}(v)=\{\ell, b, r, t\}$ and $\mathcal{C}(w)=\{L, B, R, T\}$. After possible renaming we may assume $t>T$. Note that the constructed representation is horizontally symmetric, so up to symmetry, we may assume that $\mathcal{C}(v)$ is an I and $s(v)$ is the downward diagonal of $Q(v)$.

If $r<L$ then $s(v)$ resides in the halfspace $\{x \leq r\}$ and $s(w)$ resides in the halfspace $\{x \geq L\}$ and they do not intersect. If $b>T$, then similarly $s(v)$ resides in the halfspace $\left\{y \geq 2^{b}\right\}$ while $s(w)$ resides in the halfspace $\left\{y \leq 2^{T}\right\}$, and they do not intersect. So we know that $L<r$ and $b<T<t$.

The horizontal segment of $\mathcal{C}(w)$ is $(L, T)-(R, T)$. If $R>r$, then this intersects the vertical segment $(r, b)-(r, t)$ of $\mathcal{C}(v)$, and $(v, w)$ is an edge and we are done.

The only remaining case is hence $\max \{L, R\}<r$ and $b<T<t$. Recall that $s(w)$ resides within $Q(w)=[L, R] \times\left[(2 n)^{B},(2 n)^{T}\right]$. We claim that $s(v)$ does not intersect $Q(w)$. Namely, $s(v)$ (which is the downward diagonal) has slope

$$
-\frac{(2 n)^{t}-(2 n)^{b}}{r-\ell}<-\frac{(2 n)^{t}}{2 n}=-(2 n)^{t-1} \leq-(2 n)^{T}
$$

which means that at $x$-coordinate $r-1$, the $y$-coordinate of $s(v)$ is at least $(2 n)^{b}+(2 n)^{T}>$ $(2 n)^{T}$. Since $r-1 \geq R$, this means that $s(v)$ bypasses $Q(w)$ entirely, and in particular $s(v)$ does not intersect $s(w)$.

Lemma 4. If $(v, w)$ is an edge, then $s(v)$ and $s(w)$ intersect.

Proof. Let $\mathcal{C}(v)=\{\ell, b, r, t\}$ and $\mathcal{C}(w)=\{L, B, R, T\}$. After possible renaming we may assume that the intersection of the curves happens between the vertical segment of $v$ and the horizontal segment of $w$. Up to symmetry, we may assume that $\mathcal{C}(v)$ is an $\mathrm{T}$, so $s(v)$ is the downward diagonal. This implies $L<r<R$ and $b<T<t$.

Consider the two vertical lines $\mathcal{L}_{r-1}:\{x=r-1\}$ and $\mathcal{L}_{r}:\{x=r\}$; these intersect both $s(v)$ and $s(w)$ by $\ell<r$ and $L<r<R$. As in the previous proof, one shows that $s(v)$ has $y$-coordinate $>(2 n)^{T}$ at $x=r-1$, while $s(w)$ has $y$-coordinate $\leq(2 n)^{T}$ throughout, so $s(w)$ intersects $\mathcal{L}_{r-1}$ below $s(v)$.

Segment $s(v)$ intersects $\mathcal{L}_{r}$ at $y$-coordinate $(2 n)^{b}$ and ends here. (See e.g. vertex $b$ in Figure 2.) If $s(w)$ is the downward diagonal, then it intersects $\mathcal{L}_{r}$ at $y$-coordinate

$$
(2 n)^{B}+(R-r) \frac{(2 n)^{T}-(2 n)^{B}}{R-L}=\frac{r-L}{R-L}(2 n)^{B}+\frac{R-r}{R-L}(2 n)^{T}>\frac{1}{2 n}(2 n)^{T}=(2 n)^{T-1} \geq(2 n)^{b},
$$


so it is above $s(v)$ at $\mathcal{L}_{r}$. (See e.g. vertex $c$ in Figure 2.) If $s(w)$ is the upward diagonal, then it intersects $\mathcal{L}_{r}$ at $y$-coordinate

$$
(2 n)^{T}-(R-r) \frac{(2 n)^{T}-(2 n)^{B}}{R-L}=\frac{r-L}{R-L}(2 n)^{T}+\frac{R-r}{R-L}(2 n)^{B}>\frac{1}{2 n}(2 n)^{T} \geq(2 n)^{b},
$$

so again it is above $s(v)$ at $\mathcal{L}_{r}$. (See e.g. vertex $a$ in Figure 2.) Either way therefore, somewhere in the $x$-range $[r-1, r]$ the two line segments intersect.

\section{Conclusion}

While we have made progress on one interesting question (what resolution is needed for segment representations of planar graphs?), we leave many questions open for future study:

- Is exponential resolution ever needed for planar graphs? Put differently, can we construct a planar graph $G$ such that any segment representation of $G$ requires resolution $\Omega\left(c^{n}\right)$, for some constant $c$ ? Or can we achieve polynomial resolution?

The problem is also interesting for the superclass of "graphs that have an L-representation".

- Can we achieve exponential resolution for all graphs with an \{I, L\}-representation?

- Can we construct an explicit graph family that has segment representations, but no such segment representation has exponential resolution?

- Does every graph with a segment representation have one of resolution $f(n)$, for some computable function $f(n)$ ?

\section{References}

[1] J. Chalopin and D. Gonçalves. Every planar graph is the intersection graph of segments in the plane: extended abstract. In ACM Symposium on Theory of Computing (STOC 2009), pages 631-638, 2009.

[2] Daniel Gonçalves, Lucas Isenmann, and Claire Pennarun. Planar graphs as Lintersection or L-contact graphs. In SIAM Symposium on Discrete Algorithms (SODA 2018), pages 172-184, 2018.

[3] Jan Kratochvíl. String graphs II. Recognizing string graphs is NP-hard. J. Comb. Theory, Ser. B, 52(1):67-78, 1991.

[4] Jan Kratochvíl. A special planar satisfiability problem and a consequence of its NPcompleteness. Discrete Applied Mathematics, 52(3):233-252, 1994.

[5] Jan Kratochvíl and Jiří Matoušek. String graphs requiring exponential representations. J. Comb. Theory, Ser. B, 53(1):1-4, 1991. 
[6] Jiří Matoušek. Intersection graphs of segments and $\exists \mathbb{R}$. CoRR, abs/1406.2636, 2014.

[7] Matthias Middendorf and Frank Pfeiffer. The max clique problem in classes of stringgraphs. Discrete Mathematics, 108(1-3):365-372, 1992.

[8] M. Schaefer, E. Sedgwick, and D. Stefanovic. Recognizing string graphs is in NP. Journal of Comput. Syst. Sci., 67(2):365-380, 2003.

[9] Marcus Schaefer. Crossing Numbers of Graphs. CRC Press, 2017.

[10] Edward R. Scheinerman. Intersection Classes and Multiple Intersection Parameters of Graphs. PhD thesis, Princeton University, 1984. 

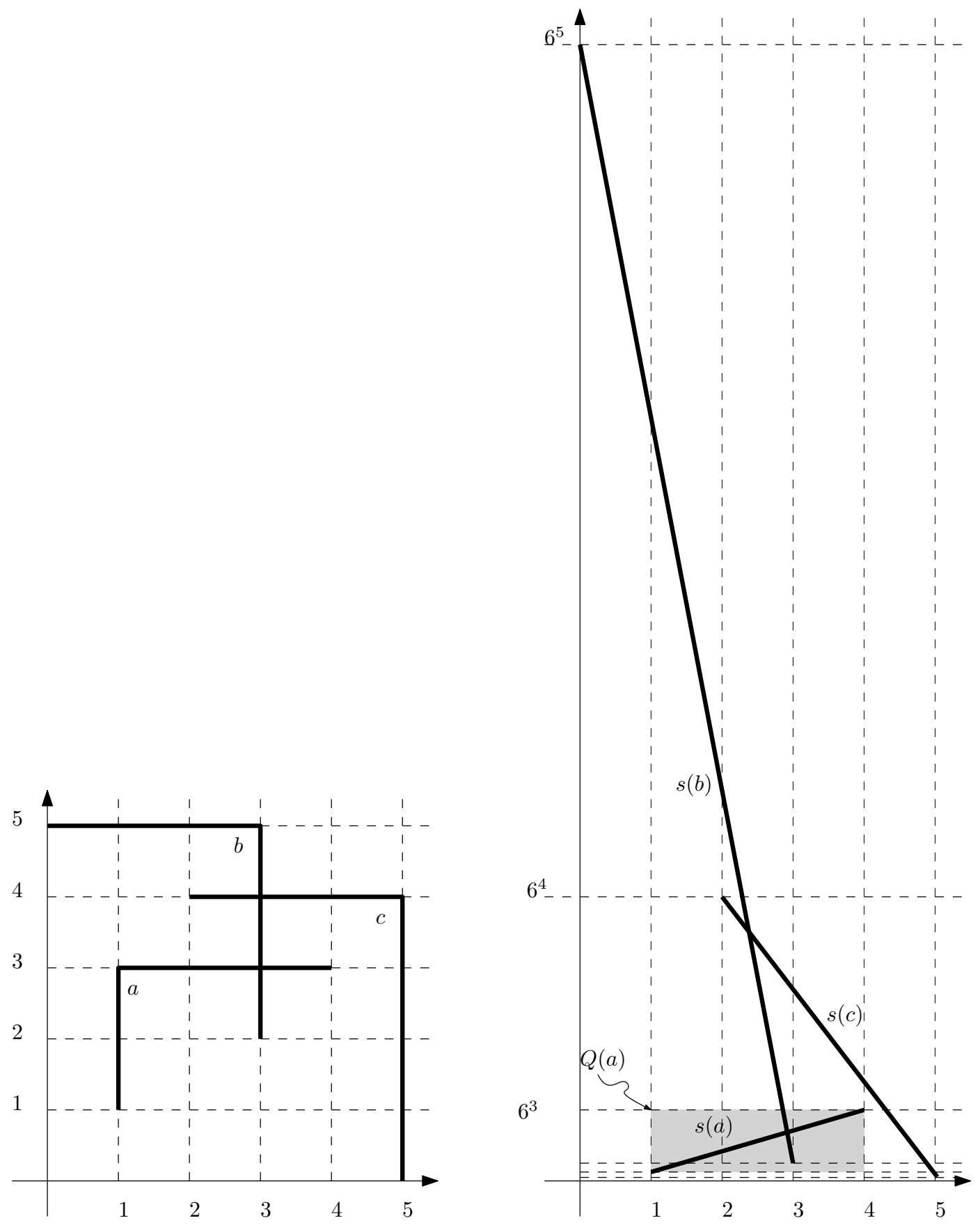

Figure 2: An $\{\mathrm{L}, \mathrm{I}\}$-representation (rotated) and its corresponding segment representation (not to scale).

8 